ANNALES

POLONICI MATHEMATICI

$87(2005)$

\title{
Feuilletages orthogonaux du tore à feuilles fermées
}

\author{
par JeAn-Marie LiOn (Rennes)
}

À la mémoire de Stanisław Łojasiewicz et de René Thom

\begin{abstract}
We give examples of pairs of orthogonal vector fields with closed orbits in every homotopy class of pairs of transversal vector fields on a two-dimensional riemannian torus.
\end{abstract}

1. Introduction. On considère sur un tore $(\mathbb{T}, g)$ de dimension 2 muni d'une métrique riemannienne de classe $C^{k}, k=2, \ldots, \infty, \omega$, une paire de feuilletages transverses $\left(\mathcal{F}_{1}, \mathcal{F}_{2}\right)$ à feuilles toutes fermées. On associe à chaque feuilletage $\mathcal{F}_{i}$ la famille $\mathcal{X}_{i}$ des champs de vecteurs sans singularité dont les orbites sont les feuilles de $\mathcal{F}_{i}$. La famille $\mathcal{X}_{i}$ est bien sûr non vide et si $X \in \mathcal{X}_{i}$ alors $\mathcal{X}_{i}$ est égale à la famille des champs $f X$ avec $f: \mathbb{T} \rightarrow \mathbb{R}, C^{k}$ et sans zéro.

On définit la relation d'équivalence suivante. Deux paires $\left(\mathcal{F}_{1}, \mathcal{F}_{2}\right)$ et $\left(\mathcal{F}_{1}^{\prime}, \mathcal{F}_{2}^{\prime}\right)$ sont équivalentes s'il existe une isotopie $H:[0,1] \times T \rightarrow T$ qui permet de passer de la première à la seconde paire. La classe d'équivalence d'une paire $\left(\mathcal{F}_{1}, \mathcal{F}_{2}\right)$ est caractérisée par la classe d'isotopie d'une paire $\left(V_{1}, V_{2}\right)$ constituée d'une feuille de chacun des deux feuilletages. Puisque $\mathcal{F}_{1}$ et $\mathcal{F}_{2}$ sont des feuilletages non singuliers et transverses de $\mathbb{T}, V_{1}$ et $V_{2}$ sont homotopiquement non triviales et ne sont pas homotopes. L'objectif de ce texte est de répondre à une question de Guy Métivier en montrant qu'une paire de feuilletages transverses $\left(\mathcal{F}_{1}, \mathcal{F}_{2}\right)$ à feuilles toutes fermées est toujours équivalente à une paire de feuilletages orthogonaux à feuilles toutes fermées.

ThÉorÈme. Soit $(\mathbb{T}, g)$ un tore $\mathbb{T}$ muni d'une métrique $g$ de classe $C^{k}$, $k=2, \ldots, \infty, \omega$, et $\gamma_{1}, \gamma_{2}$ deux courbes fermées simples de $\mathbb{T}$, non homotopes et non homotopiquement triviales. Il existe sur $\mathbb{T}$ deux feuilletages $C^{k}$, non singuliers, orthogonaux dont les feuilles sont des courbes fermées homotopes $\grave{a} \gamma_{1}$ ou $\gamma_{2}$.

2000 Mathematics Subject Classification: 37C10, 57R25, 14P15.

Key words and phrases: vector field, torus, riemannian metric, analytic geometry, isothermal coordinates, closed orbit. 
Ce théorème doit beaucoup à Dominique Cerveau. Il m'a parlé de la question de Guy Métivier, puis nous avons eu de nombreuses discussions qui m'ont aidé à trouver la démonstration qui suit. Je le remercie très sincèrement.

On utilise le langage classique des feuilletages qu'on peut trouver dans [Go] par exemple.

2. Preuve du théorème. Observons qu'on peut se restreindre au cas d'un tore plat. En effet, notre problème ne dépend pas totalement de la métrique $g$ mais seulement de sa classe conforme. Or le théorème d'existence de coordonnées isothermes (Gauss dans le cas analytique, Ahlfors et Bers dans des cas plus généraux; voir [Ha] par exemple) assure que tout tore est conformément équivalent à un tore plat. Cet argument ne vaut qu'en dimension deux.

Un tore plat est le quotient de $\mathbb{R}^{2}$ muni de la métrique euclidienne par un réseau et la classification conforme des tores plats se fait par leurs modules. Aussi, par homotopie et similitude on peut supposer :

- L'axe $\mathbb{R} \mathbf{u}$ (avec $\mathbf{u}=(1,0)$ ) est une composante connexe du relevé de $\gamma_{1}$ dans $\mathbb{R}^{2}$.

- Le tore $\mathbb{T}$ est le quotient de $\mathbb{R}^{2}$ par $\mathbb{Z} \mathbf{u}+\mathbb{Z} \mathbf{v}$ avec $\mathbf{v}=(\lambda, \mu)$ où $0 \leq \lambda<1$ et $\mu>0$.

- Une composante connexe du relevé de $\gamma_{2}$ est la droite $\mathbb{R}(p \mathbf{u}+q \mathbf{v})$ avec $p \in \mathbb{Z}, q \in \mathbb{N}^{*}$ et $p+\lambda q \geq 0$. La courbe $\gamma_{2}$ s'enroule $p$ fois parallèlement à $\mathbf{u}$ et $q$ fois parallèlement à $\mathbf{v}$.

On représente dans la figure 1 les traces des relevés de $\gamma_{1}$ et $\gamma_{2}$ dans un domaine fondamental.

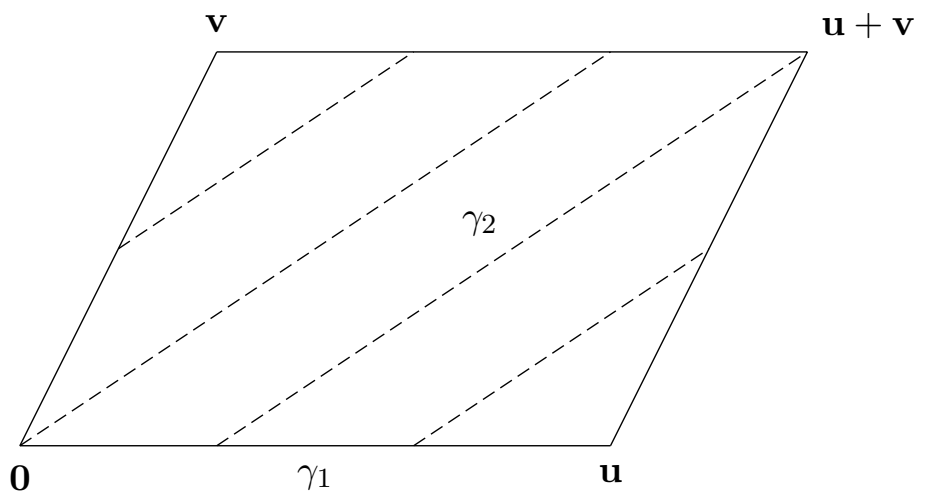

Fig. 1. Domaine fondamental, $\gamma_{1}$ et $\gamma_{2}$ avec $p=2$ et $q=3$

On va rechercher une solution parmi les paires de feuilletages orthogonaux sur le tore qui se relèvent en des paires de feuilletages orthogonaux de $\mathbb{R}^{2}$, 
invariants sous l'action du groupe de Lie des translations dirigées par les vecteurs de la droite $\mathbb{R}(r \mathbf{u}+\mathbf{v})$ (où $r \in \mathbb{N}$ est fixé plus loin) et invariants sous l'action des translations de vecteurs $\mathbf{u}$ et $\mathbf{v}$.

Si $p+\lambda q=0$ alors les feuilletages en droites horizontales et verticales induisent sur le tore une paire de feuilletages solution.

On suppose dorénavant que $p+\lambda q>0$.

On note $\mathcal{F}$ le feuilletage affine de $\mathbb{R}^{2}$ en droites parallèles à $p \mathbf{u}+q \mathbf{v}$ et de pente euclidienne égale à $q \mu /(p+\lambda q)$. Il passe au quotient en un feuilletage $\mathcal{F}_{\mathbb{T}}$ du tore dont les feuilles sont des courbes fermées homotopes à $\gamma_{2}$. En revanche, généralement les orbites de l'unique feuilletage orthogonal à $\mathcal{F}_{\mathbb{T}}$ ne sont pas fermées.

Soit $r \in \mathbb{N}$ tel que $\mu /(r+\lambda)<q \mu /(p+\lambda q)$. Soient $\delta^{+}$et $\delta^{-}$les droites dirigées par $r \mathbf{u}+\mathbf{v}$ et qui passent respectivement par les points $\mathbf{v} / r$ et 0 . Le feuilletage affine $\mathcal{F}$ est transverse à $r \mathbf{u}+\mathbf{v}$. On note $h: \delta^{+} \rightarrow \delta^{-}$l'holonomie associée à $\mathcal{F}$ qui à $x \in \delta^{+}$associe l'unique point de $\delta^{-}$qui est sur la même feuille de $\mathcal{F}$. C'est une translation vers la gauche : il existe $\tau<0$ tel que si $\left(x_{1}, x_{2}\right) \in \delta^{+}$alors $h(x)=\left(y_{1}, y_{2}\right)$ avec $y_{1}=\tau+x_{1}$.

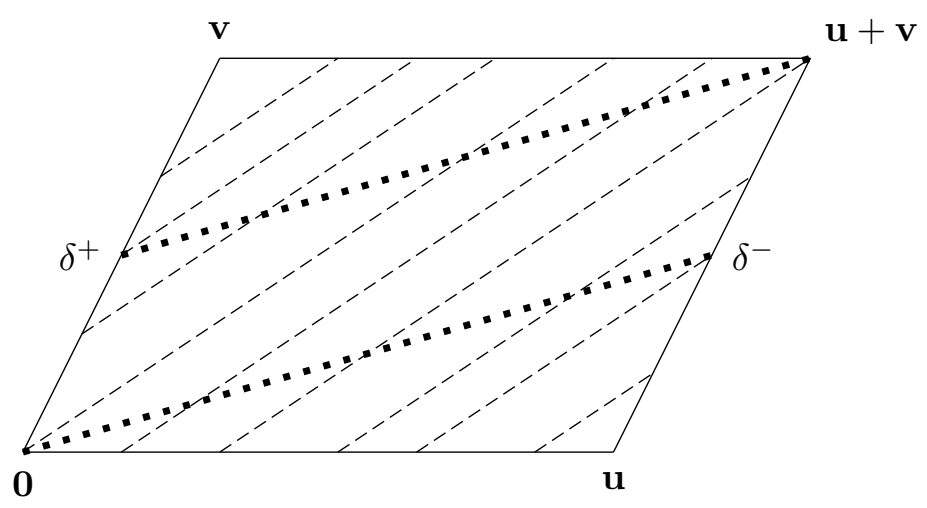

Fig. 2. Feuilles de $\mathcal{F}$ et les droites $\delta^{+}, \delta^{-}$avec $p=2, q=3$ et $r=2$

Nous allons construire notre exemple de la façon suivante. Le feuilletage horizontal est invariant sous l'action du groupe de Lie des translations dirigées par les vecteurs de la droite $\mathbb{R}(r \mathbf{u}+\mathbf{v})$ et des translations de vecteurs $\mathbf{u}$ et $\mathbf{v}$. Il passe au quotient en un feuilletage du tore dont les feuilles sont des courbes fermées homotopes à $\gamma_{1}$. Nous allons le déformer continûment en préservant cette invariance pour obtenir un feuilletage dont le feuilletage orthogonal aura la même holonomie que $\mathcal{F}$.

On considère une fonction $f: \mathbb{R} \rightarrow \mathbb{R}$, analytique, 1-périodique et qui vérifie :

(1) si $x \in[0,1 / 2[\cup] 1 / 2,1],-(r+\lambda) / \mu<f^{\prime}(x)<\mu /(r+\lambda)$, 
(2) $f(1 / 2)=0$ et $f^{\prime}(1 / 2)=-(r+\lambda) / \mu$.

Par exemple si $\lambda=0, \mu=2$ et $0<p / q<r=1$ alors $f(x)=\sin (2 \pi x) / 4 \pi$ convient.

On déduit de (1) que le graphe de $\varepsilon f$ est transverse aux droites parallèles à $r \mathbf{u}+\mathbf{v}$ et n'est jamais orthogonal à ces droites si $\varepsilon \in[0,1[$. En revanche, le graphe de $f$ est partout transverse aux droites parallèles à $r \mathbf{u}+\mathbf{v}$ mais il est orthogonal à la direction $r \mathbf{u}+\mathbf{v}$ en $(1 / 2,0)$ d'après $(2)$. On associe donc à la fonction analytique $\varepsilon f$ un feuilletage analytique $\mathcal{G}_{\varepsilon}$ de $\mathbb{R}^{2}$ défini de la façon suivante. Ses feuilles sont les translatés du graphe de $\varepsilon f$ parallèlement à $r \mathbf{u}+\mathbf{v}$. Par passage au quotient on obtient sur le tore $\mathbb{T}$ un feuilletage analytique, non singulier $\mathcal{G}_{\mathbb{T} \varepsilon}$ dont les feuilles sont en raison de la périodicité de $f$ des courbes fermées homotopes à $\gamma_{1}$. On note $\mathcal{G}_{\varepsilon}^{\perp}$ le feuilletage orthogonal à $\mathcal{G}_{\varepsilon}$. Le feuilletage $\mathcal{G}_{\mathbf{T}} \stackrel{\perp}{ }$ obtenu par passage au quotient est orthogonal à $\mathcal{G}_{\mathbb{T} \varepsilon}$.

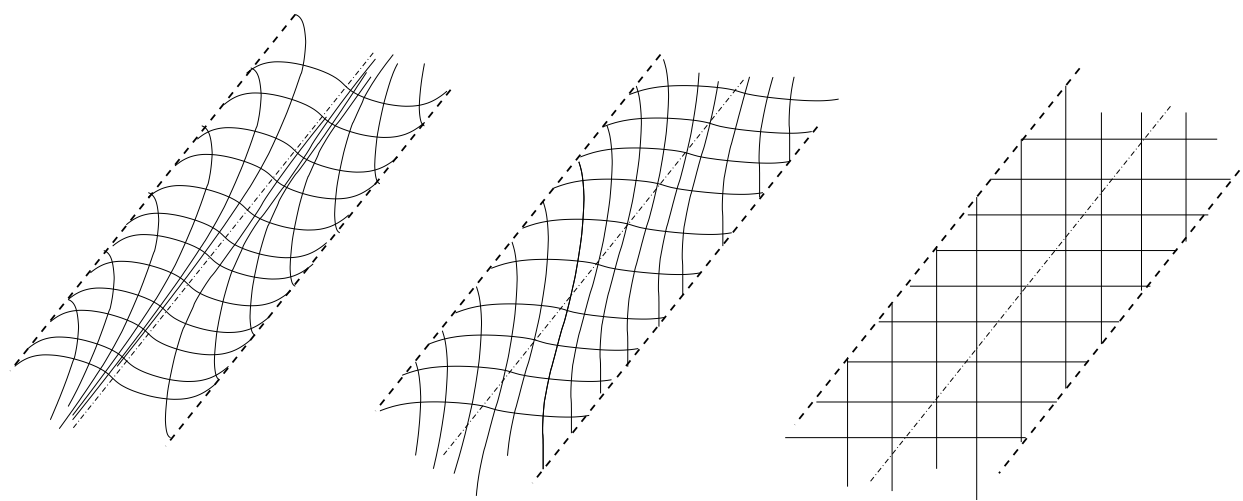

Fig. 3. Les feuilles de $\mathcal{G}_{\mathbb{T}_{\varepsilon}}$ et $\mathcal{G}_{\mathbf{T} \varepsilon}^{\perp}$ entre $\delta^{+}$et $\delta^{-}$lorsque $\varepsilon=1,0<\varepsilon<1$ et $\varepsilon=0$

Montrons qu'on peut choisir $\varepsilon \in[0,1$ [ de telle sorte que les feuilles du feuilletage $\mathcal{G}_{\overline{\mathbf{T}} \varepsilon}^{\perp}$ soient des courbes fermées homotopes à $\gamma_{2}$.

D'après (1), si $\varepsilon \in\left[0,1\left[\right.\right.$, le feuilletage $\mathcal{G}_{\varepsilon}$ n'est jamais orthogonal aux droites parallèles à $r \mathbf{u}+\mathbf{v}$ et donc le feuilletage orthogonal $\mathcal{G}_{\varepsilon}^{\perp}$ est transverse à ces droites. De plus, chaque feuille de $\mathcal{G}_{\varepsilon}^{\perp}$ coupe une et une seule fois toute droite parallèle à $r \mathbf{u}+\mathbf{v}$. L'application d'holonomie $h_{\varepsilon}: \delta^{+} \rightarrow \delta^{-}$associée à $\mathcal{G}_{\varepsilon}^{\perp}$ est donc bien définie : c'est l'application qui à chaque point de $\delta^{+}$associe l'unique point de $\delta^{-}$qui est sur la même feuille de $\mathcal{G}_{\varepsilon}^{\perp}$. C'est une translation car $\mathcal{G}_{\varepsilon}^{\perp}$ est invariant sous l'action de $\mathbb{R}(r \mathbf{u}+\mathbf{v})+\mathbb{Z} \mathbf{v} / r$ : il existe $a_{\varepsilon} \in \mathbb{R}$ tel que si $\left(x_{1}, x_{2}\right) \in \delta^{+}$alors $h_{\varepsilon}(x)=\left(y_{1}, y_{2}\right)$ avec $y_{1}=a_{\varepsilon}+x_{1}$. La fonction $\varepsilon \in\left[0,1\left[\mapsto a_{\varepsilon}\right.\right.$ est continue, $a_{0}=0$ et en raison de $(2)$ on a $\lim _{\varepsilon \rightarrow 1^{-}} a_{\varepsilon}=-\infty$. Ainsi $a_{\varepsilon}$ décrit tout l'intervalle ] $\left.-\infty, 0\right]$ (et peut-être plus), lorsque $\varepsilon$ décrit l'intervalle $\left[0,1\left[\right.\right.$. On choisit $\varepsilon$ tel que $a_{\varepsilon}=\tau$. Dans ce cas $h_{\varepsilon}=h$. Puisque $\mathcal{G}_{\varepsilon}^{\perp}$ et $\mathcal{F}$ sont invariants sous l'action de $\mathbb{R}(r \mathbf{u}+\mathbf{v})+\mathbb{Z} \mathbf{v} / r$, les feuilles de $\mathcal{G}_{\mathbf{T}}^{\perp}$ sont homotopes à celles de $\mathcal{F}_{\mathbb{T}}$ : ce sont des courbes fermées homotopes à $\gamma_{2}$. 
3. Une famille explicite. On conserve les notations de la partie précédente. Les données sont donc $\mathbf{u}, \mathbf{v}, \lambda, \mu, p, q$ et un entier $r$ tel que $\mu /(r+\lambda)$ $<q \mu /(p+\lambda q)$. On pose $\alpha=\mu /(r+\lambda)$. On définit alors sur $\mathbb{R}^{2}$ les deux familles de champs de vecteurs

$$
\begin{aligned}
X_{\varepsilon}= & \left(1-\frac{\varepsilon}{1+\alpha^{2}} \cos (2 \pi(x-y / \alpha))\right) \frac{\partial}{\partial x}-\frac{\varepsilon \alpha}{1+\alpha^{2}} \cos (2 \pi(x-y / \alpha)) \frac{\partial}{\partial y} \\
Y_{\varepsilon}= & \frac{\varepsilon \alpha}{1+\alpha^{2}} \cos (2 \pi(x-y / \alpha)) \frac{\partial}{\partial x} \\
& +\left(1-\frac{\varepsilon}{1+\alpha^{2}} \cos (2 \pi(x-y / \alpha))\right) \frac{\partial}{\partial y}=X_{\varepsilon}^{\perp}
\end{aligned}
$$

où $\varepsilon \in[0,1]$.

À $\varepsilon$ fixé, ces deux champs sont orthogonaux, invariants sous l'action $\mathrm{du}$ groupe de Lie des translations dirigées par les vecteurs de la droite $\mathbb{R}(r \mathbf{u}+\mathbf{v})$ et invariants sous l'action des translations de vecteurs $\mathbf{u}$ et $\mathbf{v}$. Un petit calcul montre que si $\varepsilon \neq 1$ les champs $X_{\varepsilon}$ et $Y_{\varepsilon}$ sont transverses aux droites parallèles à $r \mathbf{u}+\mathbf{v}$. Le champ $X_{1}$ est lui aussi transverse à ces droites. Ce n'est pas le cas du champ $Y_{1}$, qui est parallèle à la direction $r \mathbf{u}+\mathbf{v}$ le long des droites du type $\mathbb{R}(r \mathbf{u}+\mathbf{v})+k \mathbf{v} / r, k \in \mathbb{Z}$, qui sont $Y_{1}$-invariantes. Par passage au quotient ils définissent des paires de champs de vecteurs orthogonaux sur le tore $\mathbb{T}$. Il existe une fonction $f$ associée à $X_{1}$ comme dans la partie précédente et pour chaque $\varepsilon \in[0,1]$ il existe une fonction $f_{\varepsilon}$ associée à $X_{\varepsilon}$.

La situation est différente de celle de la partie précédente car maintenant $f_{\varepsilon}$ ne s'exprime pas très facilement en fonction de $f$, c'est seulement $X_{\varepsilon}$ qui dépend affinement de $\varepsilon$. Cependant, en faisant une analyse semblable à celle de la partie 2 on montre qu'il existe $\varepsilon$ tel que la paire de feuilletages du tore associée à la paire $\left(X_{\varepsilon}, Y_{\varepsilon}\right)$ vérifie les conclusions du théorème. L'intérêt de cet exemple explicite est qu'il est intégrable. Si on se restreint au traces des solutions dans le domaine fondamental engendré par les directions $\mathbf{u}$ et $\mathbf{v}$, alors les solutions appartiennent à la clôture pfaffienne [Sp] de la structure o-minimale engendrée par la restriction de la fonction sinus à l'intervalle $[0,2 \pi]$.

4. Une petite question de Ludwig Bröcker. Pour finir, je mentionne une jolie question que Ludwig Bröcker a posée à la suite de mon exposé de mars 2004 au colloque RAAG de Cracovie. Il demande si on peut préciser le théorème de la façon suivante. Étant donnés $\mathbb{T}, \gamma_{1}, \gamma_{2}$, est-ce que la famille des paires $\left(\mathcal{F}_{1}, \mathcal{F}_{2}\right)$ vérifiant les conclusions du théorème est connexe? À ma connaissance le problème reste ouvert, même si on se limite aux paires $\left(\mathcal{F}_{1}, \mathcal{F}_{2}\right)$ qui se relèvent en des paires invariantes sous l'action du groupe de Lie des translations dirigées par les vecteurs de la droite $\mathbb{R}(r \mathbf{u}+\mathbf{v}$ ) (avec $r \in \mathbb{N}$ ad hoc) et sous l'action des translations de vecteurs $\mathbf{u}$ et $\mathbf{v}$. 


\section{Bibliographie}

[Go] C. Godbillon, Feuilletages. Études géométriques, Progr. Math. 98, Birkhäuser, Basel, 1991.

[Ha] P. Haïssinsky, Définition de la quasi-conformité, prépublication, 2001.

[Sp] P. Speissegger, The Pfaffian closure of an o-minimal structure, J. Reine Angew. Math. 508 (1999), 189-211.

IRMAR, UMR 6625 CNRS

Université Rennes 1

35042 Rennes Cedex, France

E-mail: jean-marie.lion@univ-rennes1.fr

Reçu par la Rédaction le 12.10.2004 